\title{
Minimizing right ventricular pacing in patients with sinus node disease and prolonged $P Q$ interval: The impact on exercise capacity
}

\author{
Krystian Krzyżanowski ${ }^{1}$, Dariusz Michałkiewicz ${ }^{2}$, Zbigniew Orski ${ }^{1}$, \\ Robert Wierzbowski ${ }^{1}$, Robert Ryczek ${ }^{1}$, Andrzej Cwetsch ${ }^{1}$ \\ ${ }^{1}$ Department of Cardiology and Internal Diseases, \\ Military Institute of Medicine, Warsaw, Poland \\ ${ }^{2}$ Military Institute of Medicine, Warsaw, Poland
}

\begin{abstract}
Background: Several clinical and experimental studies have shown that unnecessary right ventricular pacing in sinus node disease can be detrimental. Inter-and intra-ventricular asynchrony imposed by right ventricular pacing may cause reduction in contractility and relaxation of left ventricle, worsening mitral regurgitation, regional redistribution of myocardial perfusion and oxygen consumption, and asymmetrical hypertrophy of left ventricular wall. In some patients, sinus node disease coexists with impaired atrioventricular conduction. The optimal pacing mode in this population is not determined. Minimizing right ventricular pacing can preserve inter- and intra-ventricular synchrony. On the other hand, longer atrioventricular delay may cause atrioventricular asynchrony. The aim of this study was to prospectively assess the impact of minimizing right ventricular pacing in patients with DDD pacemaker implanted for sinus node disease and prolonged $P Q$ interval on exercise capacity, cardiac function and quality of life.
\end{abstract}

Methods: The study enrolled 50 consecutive patients with prolonged PQ interval who were implanted with DDD pacemaker because of sinus node disease. Each patient was treated alternately with 2 pacing modes in random order for 4-month periods: conventional dual-chamber pacing and dual-chamber minimal ventricular pacing (prolonged atrioventricular delay). At the end of each phase the following tests were performed: cardiopulmonary exercise testing, echocardiographic evaluation and quality of life assessment (SF36).

Results: There was no significant relationship between pacing mode and cardiopulmonary parameters, echocardiographic parameters and quality of life.

Conclusions: Sequential atrioventricular pacing may be a reasonable choice for treating patients with sinus node disease and prolonged PQ interval. (Cardiol J 2015; 22, 1: 75-79)

Key words: sick sinus syndrome, first degree atrioventricular block, pacing

Address for correspondence: Krystian Krzyżanowski, MD, PhD, Department of Cardiology and Internal Diseases, Military Institute of Medicine, ul. Szaserów 128, 04-141 Warszawa, Poland, tel: +48 512356 207,

e-mail: krystian.krzyzanowski@gmail.com

Received: 22.02.2014 Accepted: 27.03.2014 


\section{Introduction}

Several clinical and experimental studies have shown that unnecessary right ventricular pacing (RVP) in sinus node disease (SND) can be detrimental. Inter- and intra-ventricular asynchrony imposed by RVP may cause reduction in contractility and relaxation of left ventricle (LV), worsening mitral regurgitation, regional redistribution of myocardial perfusion and oxygen consumption, and asymmetrical hypertrophy of the LV wall [1-7].

In $23-58 \%$ of patients with SND, atrioventricular (AV) conduction is also impaired. Two thirds of these patients have first degree AV block [8, 9]. Prolonged AV conduction may lead to diastolic mitral regurgitation and in some patients may decrease exercise capacity $[10,11]$.

The optimal pacing mode in patients with SND and concomitant first degree AV block is not determined. Minimizing RVP can preserve interand intra-ventricular synchrony. On the other hand, longer AV delay may cause AV asynchrony. The purpose of the present study was to assess the impact of minimizing RVP on exercise capacity, cardiac function and quality of life.

\section{Methods}

The study was approved by the Institutional Review Board of the Military Institute of Medicine (IRB followed the Helsinki recommendations, approval number $32 / \mathrm{WIM} / 2008$ ). All of the subjects provided informed consent.

We prospectively assessed patients that had had an implantation of DDD pacemaker in our hospital due to SND. The inclusion criteria were: (1) PQ interval $\geq 200 \mathrm{~ms}$ at baseline; (2) the percentage of RVP $\geq 90 \%$ during the $1^{\text {st }}$ week after the procedure (with default AV delay). Patients were excluded if they had second or third degree AV block or severely impaired LV systolic function (LV ejection fraction $[\mathrm{LVEF}] \leq 35 \%$ ). Patients eligible for the study were included on the $7^{\text {th }}$ day after implantation. The study had a crossover design. Every patient was observed for 2 consecutive periods lasting $3-5$ months. In the $1^{\text {st }}$ of the study periods standard $\mathrm{AV}$ delay was set. In the other, $\mathrm{AV}$ delay was prolonged to achieve minimized ventricular pacing. The order of these time periods was randomized. After the completion of the $1^{\text {st }}$ period every subject was treated with the opposite AV delay settings for approximately the same time. At the end of each study period the following data was acquired: (1) percentage of ventricular and atrial pacing (extracted from the pacemaker storage); (2) exercise capacity assessed by cardiopulmonary exercise testing (CPET); (3) LV and left atrial dimensions and LVEF determined by echocardiography; (4) quality of life assessed by short form 36 questionnaire (SF36). A paired approach was adopted in the analysis of the data.

\section{Pacemaker settings}

The exact AV delay settings were individualized and dependent on a model of pacemaker, baseline $\mathrm{PQ}$ interval, and upper tracking rate. The maximal AV delay (for the lower rate) in the standard period was $180 \mathrm{~ms}$, minimal: $170 \mathrm{~ms}$ ( 177 ms on average). In the minimized RVP period the numbers were $350 \mathrm{~ms}$ and $250 \mathrm{~ms}$, respectively (301 ms on average). Automatic shortening of the AV delay was allowed during rate increases. The lower rate during the diurnal and nocturnal hours was set at $60 \mathrm{bpm}$ and $50-55 \mathrm{bpm}$, respectively. The upper rate was set according to age and general condition of each patient. In the case of chronotropic incompetence, the rate response features were adopted. The above mentioned settings, except for the AV delays, were not changed during the whole study period.

\section{Cardiopulmonary exercise testing}

Symptom-limited CPET was performed on a cycle or treadmill ergometer with a protocol that was personalized with the aim of having each patient reach maximum exercise in 7-10 min. The protocol was the same for both study arms. Breath-by-breath measurements of gas exchange were obtained by a metabolic cart (ZAN 680; ZAN Messgerate $\mathrm{GmbH}$; Germany). Minute ventilation (VE), oxygen uptake and carbon dioxide output $\left(\mathrm{VCO}_{2}\right)$ were averaged over $10 \mathrm{~s}$. Peak oxygen uptake $\left(\mathrm{VO}_{2}\right)$ was expressed as the mean value of $\mathrm{VO}_{2}$ during the last $30 \mathrm{~s}$ of the exercise test. Ventilatory anaerobic threshold (VAT) was detected by the $\mathrm{V}$-slope method. $\mathrm{VE} / \mathrm{VCO}_{2}$ slope was calculated as a linear regression function using the data points from the whole exercise period. Peak oxygen pulse $\left(\mathrm{O}_{2}\right.$ pulse $)$ was delineated as peak $\mathrm{VO}_{2}$ divided by peak heart rate.

\section{Echocardiography}

All patients underwent comprehensive transthoracic echocardiography examination by system Vivid 7 GE. End-systolic and end-diastolic volumes, and ejection fraction were calculated by modified biplane Simpson's method. 


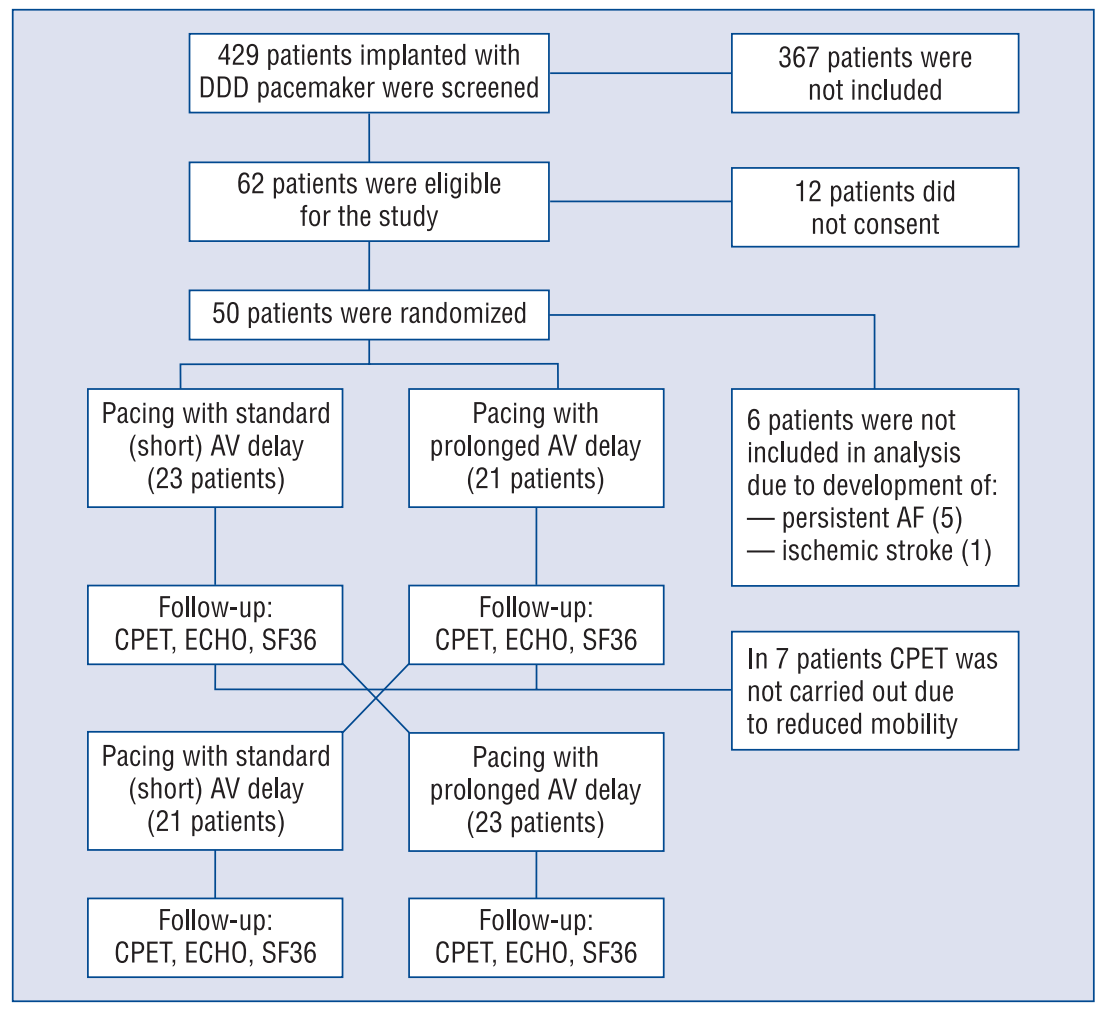

Figure 1. Flow diagram: screening, exclusion, randomization and follow-up of patients; AF - atrial fibrillation; CPET - cardiopulmonary exercise testing; ECHO - echocardiography; SF36 - short form 36 questionnaire.

\section{Statistical analysis}

Continuous data are expressed as mean \pm \pm standard deviation. A Student's paired t-test or Wilcoxon signed rank test in case of non-normally distributed data were used for repeated measures. For qualitative variables the Fisher's exact test was employed. Statistical differences with a p value $<0.05$ were considered significant. All calculations were performed with statistical software (SAS 9.3; SAS Institute Inc.; Cary, NC, USA).

\section{Results}

From June 2008 to December 2011 we enrolled 50 patients on the study. The study flow diagram is presented in Figure 1. The demographic and clinical data for 44 patients used in the final analysis are summarized in Table 1.

The mean duration of pacing with standard AV delay and of minimized ventricular pacing was $124 \pm 33$ days and $121 \pm 33$ days, respectively $(\mathrm{p}=0.81)$. During the minimized ventricular pacing period the percentage of RVP was reduced to a median of $16 \%$ in comparison to $98 \%$ during standard pacing $(\mathrm{p}<0.0001)$. The $\mathrm{QRS}$ duration
Table 1. Baseline characteristics of the patients.

\begin{tabular}{lc}
\hline Age [years] & $75 \pm 7$ \\
Male gender & $28(64 \%)$ \\
History of paroxysmal AF & $19(43 \%)$ \\
Coronary artery disease & $17(39 \%)$ \\
Arterial hypertension & $33(75 \%)$ \\
PQ interval [ms] & $234 \pm 26$ \\
QRS duration [ms] & $109 \pm 18$ \\
LV ejection fraction [\%] & $60 \pm 6$ \\
LV diastolic diameter [cm] & $4.8 \pm 0.5$ \\
RVOT position of ventricular lead & $42(96 \%)$ \\
Percentage of ventricular pacing & $97.2 \pm 2.7$ \\
during the first week after & \\
implantation [\%] & \\
Medication: & \\
Beta-blocker & $21(48 \%)$ \\
ACE inhibitors & $31(71 \%)$ \\
ARB & $7(16 \%)$ \\
Antiarrhythmics (amiodarone, & $9(21 \%)$ \\
propafenone) & \\
Calcium-channel blockers & $11(25 \%)$ \\
Diuretics & $21(48 \%)$ \\
\hline
\end{tabular}

ACE - angiotensin-converting enzyme; ARB - angiotensin receptor blockers; $A F$ - atrial fibrillation; LV — left ventricular; RVOT - right ventricle outflow tract 
Table 2. Cardiopulmonary exercise testing and echocardiographic variables.

\begin{tabular}{lccc}
\hline Variable & $\begin{array}{c}\text { AV sequential pacing } \\
\text { with standard AV delay }\end{array}$ & $\begin{array}{c}\text { Minimized ventricular } \\
\text { pacing }\end{array}$ & P \\
\hline Peak oxygen consumption $[\mathrm{mL} / \mathrm{kg} / \mathrm{min}]$ & $17.1 \pm 5.9$ & $16.6 \pm 5.3$ & $0.26 \dagger$ \\
Anaerobic threshold $[\mathrm{mL} / \mathrm{kg} / \mathrm{min}]$ & $12.3 \pm 5.0$ & $12.3 \pm 4.2$ & $0.36^{*}$ \\
VE/VCO ${ }_{2}$ slope & $29.2 \pm 4.6$ & $30.1 \pm 8.1$ & $0.69^{*}$ \\
Left ventricular diastolic diameter [cm] & $5.0 \pm 0.6$ & $5.0 \pm 0.6$ & $0.64 \dagger$ \\
Left atrium diameter [cm] & $4.34 \pm 0.47$ & $4.21 \pm 0.55$ & $0.12 \dagger$ \\
Left ventricular ejection fraction [\%] & $59.3 \pm 9.6$ & $59.2 \pm 12.1$ & $0.99 \dagger$ \\
\hline
\end{tabular}

*Wilcoxon signed-rank test; †paired Student's t-test; AV — atrioventricular

Table 3. Quality of life.

\begin{tabular}{lccc}
\hline Subscales of SF36 questionnaire & $\begin{array}{c}\text { AV sequential pacing } \\
\text { with standard AV delay }\end{array}$ & $\begin{array}{c}\text { Minimized ventricular } \\
\text { pacing }\end{array}$ & P \\
\hline Physical functioning & $63.5 \pm 18.8$ & $62.4 \pm 22.5$ & $0.48^{*}$ \\
Physical role functioning & $49.4 \pm 26.7$ & $45.9 \pm 27.5$ & $0.27 \dagger$ \\
Bodily pain & $60.8 \pm 24.0$ & $61.9 \pm 25.1$ & $0.82^{*}$ \\
General health perceptions & $50.6 \pm 13.2$ & $47.3 \pm 13.9$ & $0.052^{*}$ \\
Vitality & $52.8 \pm 18.3$ & $49.6 \pm 16.7$ & $0.17^{*}$ \\
Social role functioning & $70.3 \pm 24.0$ & $64.8 \pm 22.7$ & $0.053^{*}$ \\
Emotional role functioning & $62.4 \pm 27.5$ & $61.2 \pm 28.1$ & $0.72^{*}$ \\
Mental health & $64.2 \pm 18.4$ & $63.6 \pm 17.2$ & $0.78 \dagger$ \\
\hline
\end{tabular}

*Wilcoxon signed-rank test; †paired Student's t-test; AV — atrioventricular

was also diminished: $113 \pm 26$ vs. $155 \pm 20 \mathrm{~ms}$, respectively $(\mathrm{p}<0.0001)$. However, a quarter of patients still had more than $40 \%$ of RVP during minimized ventricular pacing. There were no significant differences between the study periods in percentage of atrial pacing $(\mathrm{p}=0.38)$.

The peak oxygen uptake, ventilatory anaerobic threshold, $\mathrm{VE} / \mathrm{VCO}_{2}$ slope, $\mathrm{LV}$ diastolic diameter, left atrial diameter and LVEF did not differ according to the pacing mode (Table 2 ). When comparing the results of 8 subscales of the SF36 questionnaire, they did not reveal any significant differences between the study groups (Table 3 ).

\section{Discussion}

The present study compared minimized RVP with standard settings of AV delay in a selected group of patients with both SND and first degree AV block. The result was that no significant improvement in exercise capacity and quality of life was associated with a reduced percentage of RVP. These findings suggest that sequential AV pacing with standard AV settings may be a reasonable choice in this group of patients.
Several laboratory and clinical studies have shown that RVP may result in reduced LV systolic and diastolic function $[6,7,12]$. Due to ventricular desynchronization ventricular pacing may promote heart failure. The risk of developing heart failure grows with increasing cumulative percentage of RVP [13-15]. However, reducing ventricular pacing by extending AV delay may lead to AV desynchronization. Prolonged PQ interval or stimulus-R interval in atrial pacing may result in diastolic mitral regurgitation, and in consequence in heart failure $[10,11,16]$. Thus, the LV performance depends on both intra-ventricular and AV synchrony. Prolonged AV conduction in all patients included in the present study may explain why minimizing ventricular pacing had no impact on exercise capacity and cardiac function.

Similar observations were presented by Vardas et al. [17] in 15 patients with SND and normal AV conduction. They reported no difference in the anaerobic threshold according to the pacing mode: AAI or DDD with short AV delay [17]. The possible reason for this result may be that AV conduction during atrial pacing in patients with SND may paradoxically lengthen with increasing 
pacing rate during exercise $[18,19]$. This lack of physiological adaptation of the AV interval when exercising may be the result of the maladjustment of the adrenergic tone to the heart rate determined by the pacemaker [18].

In contrast to our findings, Wonisch et al. [20] reported improvement in CPET variables with pacing modes that minimize ventricular pacing in 17 patients with heart failure and cardioverter-defibrillator. The possible explanation of these results may lie in the fact that RVP in hearts that are impaired at the baseline leads to a greater extending of intra-ventricular desynchronization. The QRS duration (QRSd) and LV activation time due to RVP are increased when LVEF is less than $40 \%$ in comparison to hearts with preserved LV systolic function: QRSd $209 \mathrm{~ms}$ vs. $193 \mathrm{~ms}$, respectively and LV activation time $186 \mathrm{~ms}$ vs. $129 \mathrm{~ms}$, respectively [21]. In our study, however, the LV function was not impaired at baseline with mean LVEF of $60 \%$ and QRSd of $109 \mathrm{~ms}$. This may be the reason we did not observe any deleterious effects of RVP.

\section{Limitations of the study}

There are several limitations of the present study. Small number of patients included, short follow-up period in each pacing mode, and residual ventricular pacing over $40 \%$ in a quarter of patients could have mitigated the possible benefit of minimized ventricular pacing.

\section{Conclusions}

The results of the present study support the view that sequential $\mathrm{AV}$ pacing in SND patients with prolonged $\mathrm{PQ}$ interval is a safe alternative regarding exercise capacity, cardiac function and quality of life. However, the study group was too small and the follow-up period too short to assess the impact of minimizing ventricular pacing on mortality and heart failure development.

\section{Acknowledgements}

The study was supported by the National Science Center, Poland. Grant number: N N402 533539 .

Conflict of interest: Krystian Krzyżanowski is a consultant of Biotronik Company.

\section{References}

1. Delhaas T, Arts T, Prinzen FW, Reneman RS. Regional fiber stress-fiber strain as estimate of regional oxygen demand in the canine heart. J Physiol, 1994; 477: 481-496.
2. Baller D, Wolpers HG, Zipfel J, Bretschneider HJ, Hellige G. Comparison of the effects of right atrial, right ventricular apex, and atrioventricular sequential pacing on myocardial oxygen consumption and cardiac efficiency: a laboratory investigation. Pacing Clin Electrophysiol, 1988; 11: 394-403.

3. Nielsen JC, Bottcher M, Nielsen TT. Regional myocardial blood flow in patients with sick sinus syndrome randomized to long-term single chamber atrial or dual chamber pacing: Effect of pacing mode and rate. J Am Coll Cardiol, 2000; 35: 1453-1461.

4. Van Oosterhout MF, Prinzen FW, Arts T et al. Asynchronous electrical activation induces asymmetrical hypertrophy of the left ventricular wall. Circulation, 1998; 98: 588-595.

5. Karpawich PP, Rabah R, Haas JE. Altered cardiac histology following apical right ventricular pacing in patients with congenital atrioventricular block. Pacing Clin Electrophysiol, 1999; 22: 1372-1377.

6. Nahlawi M, Waligora M, Spies S, Bonow RO, Kadish AH, Goldberger JJ. Left ventricular function during and after right ventricular pacing. J Am Coll Cardiol, 2004; 44: 1883-1888.

7. Rosenqvist M, Bergfeldt L, Haga Y, Ryden J, Ryden L, Owall A. The effect of ventricular activation sequence on cardiac performance during pacing. Pacing Clin Electrophysiol, 1996; 19: 1279-1286.

8. Vallin H, Edhag O. Associated conduction disturbances in patients with symptomatic sinus node disease. Acta Med Scand, 1981; 210: 263-270.

9. Sakai Y, Imai S, Sato Y, Yagi H, Kushiro T. Clinical and electrophysiological characteristics of binodal disease. Circ J, 2006; 70: 1580-1584.

10. Ishikawa T, Sumita S, Kimura $\mathrm{K}$ et al. Critical $\mathrm{PQ}$ interval for the appearance of diastolic mitral regurgitation and optimal $\mathrm{PQ}$ interval in patients implanted with DDD pacemakers. Pacing Clin Electrophysiol, 1994; 17: 1989-1994.

11. Dulk K, Lindemans FW, Brugada P, Smeets JL, Wellens HJ. Pacemaker syndrome with AAI rate variable pacing: Importance of atrioventricular conduction properties, medication, and pacemaker programmability. Pacing Clin Electrophysiol, 1988; 11: 1226-1233.

12. Albertsen AE, Nielsen JC, Poulsen SH et al. DDD(R)-pacing, but not $\mathrm{AAI}(\mathrm{R})$-pacing induces left ventricular desynchronization in patients with sick sinus syndrome: Tissue-Doppler and 3D echocardiographic evaluation in a randomized controlled comparison. Europace, 2008; 10: 127-133.

13. Sweeney MO, Hellkamp AS, Ellenbogen KA et al. Adverse effect of ventricular pacing on heart failure and atrial fibrillation among patients with normal baseline QRS duration in a clinical trial of pacemaker therapy for sinus node dysfunction. Circulation, 2003; 107: 2932-2937.

14. Sharma AD, Rizo-Patron C, Hallstrom AP et al. Percent right ventricular pacing predicts outcomes in the DAVID Trial. Heart Rhythm, 2005; 2: 830-834.

15. Steinberg JS, Fischer A, Wang P et al. The clinical implications of cumulative right ventricular pacing in the Multicenter Automatic Defibrillator Trial II. J Cardiovasc Electrophysiol, 2005; 16: 359-365.

16. Barold SS. Indications for permanent cardiac pacing in first-degree AV block: Class I, II or III? Pacing Clin Electrophysiol, 1996; 19: 747-751.

17. Vardas PE, Simantirakis EN, Parthenakis FI, Chrysostomakis SI, Skalidis EI, Zuridakis EG. AAIR versus DDDR pacing in patients with impaired sinus node chronotropy: An echocardiographic and cardiopulmonary study. Pacing Clin Electrophysiol, 1997; 20: 1762-1768.

18. Mabo P, Cebron JP, Solnon A, Tassin A, Graindorge L, Gras D. Non-physiological increase of AV conduction time in sinus disease patients programmed in AAIR-based pacing mode. J Interv Card Electrophysiol, 2012; 35: 219-226.

19. Schwaab B, Frohlig G, Pistorius C, Schwerdt H, Schieffer H. AV conduction with atrial rate adaptive pacing in the bradycardia tachycardia syndrome. Pacing Clin Electrophysiol, 1999; 22: 1502-1509.

20. Wonisch M, Lercher P, Scherr D et al. Influence of permanent right ventricular pacing on cardiorespiratory exercise parameters in chronic heart failure patients with implanted cardioverter defibrillators. Chest, 2005; 127: 787-793.

21. Varma N. Left ventricular conduction delays induced by right ventricular apical pacing: Effect of left ventricular dysfunction and bundle branch block. J Cardiovasc Electrophysiol, 2008; 19: 114-122. 